\title{
PLANTAS MEDICINALES: UNA FARMACIA NATURAL PARA LA SALUD PÚBLICA
}

\section{MEDICINAL PLANTS: A NATURAL PUBLIC HEALTH PHARMACY}

Gunther Balarezo López

\section{Resumen}

Culturas ancestrales han utilizado las plantas como medicina, costumbre que actualmente se está perdiendo debido a la falta de transmisión de estos conocimientos de padres a hijos. A pesar de ello, en los países subdesarrollados, su consumo continúa por su accesibilidad, costos y efectividad. Sin embargo, la medicina convencional aún no la toma en cuenta por desconocimiento o por falta de una base científica que confirme sus beneficios. En tal sentido, las Escuela de Medicina deberían incluir cursos referentes a la medicina tradicional para conocer las plantas que podrian emplearse como complemento al tratamiento farmacológico.

Palabras clave: plantas medicinales, medicina tradicional, etnobotánica.

\section{Abstract}

Ancestral cultures have used plants as medicine, a custom that is currently being lost due to the lack of transmission of this knowledge from parents to children. In spite of this, in the underdeveloped countries, the consumption of plants as medicine continues because of their accessibility, costs and effectiveness. However, conventional medicine has not yet taken it into account due to lack of knowledge or a scientific basis to confirm its benefits. Therefore, medical schools should include courses related to traditional medicine to know the plants that could be used as a complement to pharmacological treatment.

Key words: medicinal plants, traditional medicine, ethnobotany. 


\section{Introducción}

Desde su aparición sobre la Tierra, los seres humanos han recurrido a la naturaleza como fuente de conocimiento y recursos. Después de miles de años de observación y experimentación, llegaron a la conclusión de que las plantas no solo servían como alimento, sino, además, tenían propiedades con efectos específicos sobre su organismo. Este conocimiento, originalmente transmitido de manera verbal de padres a hijos, ha devenido en la medicina popular. (García, 2004; Mejía, 2000).

Sin embargo, en muchos países en desarrollo ha ocurrido una pérdida importante que pone en riesgo la cadena de transmisión de este conocimiento tradicional. Por lo tanto, se hace necesario realizar esfuerzos no solo para preservar esta herencia cultural, sino también para registrar la información sobre ciertas especies que podrían ser relevantes para el desarrollo de nuevas fuentes de medicamentos $\mathrm{y}$ de otros beneficios para la humanidad. A esto se suma que la disponibilidad de dichas plantas se ha visto reducida por la degradación de los bosques y su conversión a campos agrícolas. (Tabuti, 2003;Bermúdez, 2005).

A pesar de esta disminución en la transmisión del conocimiento, el uso medicinal de las plantas y derivados con finalidad terapéutica (prevenir, aliviar o curar enfermedades), nunca ha dejado de tener vigencia, dado que los tratamientos a base de plantas presentan una ventaja con respecto a los tratamientos químicos, los cuales son capaces de producir intoxicaciones e intolerancias. En las plantas, los principios activos se hallan siempre biológicamente equilibrados por la presencia de sustancias complementarias que van a potenciarse entre sí, no acumulándose en el organismo, limitando así sus efectos indeseables. (Diario Expreso, 2015b).

Pero también se mencionan desventajas en su uso, y contrario a la creencia popular, las plantas y las preparaciones vegetales pueden causar efectos adversos y tóxicos, reacciones alérgicas severas, interacciones peligrosas con medicamentos convencionales e interferir con pruebas de laboratorio, causando riesgos para la salud. También, más allá de estos riesgos directos, existe el riesgo indirecto de que un remedio natural sin eficacia comprobada pueda comprometer, retardar o reemplazar una forma eficaz y efectiva de tratamiento convencional. Asimismo, las plantas que son aparentemente seguras bajo condiciones normales, pueden ser peligrosas en pacientes bajo condiciones de vulnerabilidad (ancianos, niños), enfermedades específicas (diabetes), circunstancias especiales (periodo perioperatorio), o cuando se combinan con medicamentos (anticoagulantes, antiretrovirales). Los efectos adversos pueden variar ampliamente y dependen de la especie particular de la planta, de cuándo y cómo es recolectada, de la parte de la planta usada y de cómo el material vegetal es procesado. (Sáenz, 2003). 
Por ello, para el buen uso de las plantas medicinales es necesario conocer correctamente las especies utilizadas, la forma de preparación y dosificación. Muchos de los compuestos presentes en las plantas actúan de modo sinérgico, de modo que la combinación de dos o más especies es condición necesaria para obtener efectos benéficos. (Mejía, 2000). De particular interés ha sido el empleo de la medicina herbolaria, que es parte de la medicina tradicional y que comprende el uso de plantas o partes de éstas en su estado natural; es decir, sin procesamiento químico. (Bocanegra, 2011).

\section{Plantas Medicinales}

De acuerdo a la Organización Mundial de la Salud (OMS), una planta medicinal se define como cualquier especie vegetal que contiene sustancias que pueden ser empleadas para propósitos terapéuticos o cuyos principios activos pueden servir de precursores para la sintesis de nuevos fármacos. La investigación sobre el uso de plantas medicinales forma parte de la etnobotánica, definida como el estudio de las interrelaciones entre los grupos humanos y las plantas y que por su naturaleza incluye áreas como: botánica, química, medicina, farmacología, toxicología, nutrición, agronomía, ecología, sociología, antropología, lingüística, historia y arqueologia, entre otras; lo cual permite un amplio rango de enfoques y aplicaciones. (Bermúdez, 2005); Gómez-Veloz, 2002; Prance, 1991).
Debido a que las plantas medicinales son el mayor recurso terapéutico usado por la medicina tradicional de muchos países, existe el interés de organismos, internacionales, como la Organización Mundial de la Salud (OMS) y la Organización Panamericana de la Salud (OPS), traducido en intervenciones básicamente de tipo promocional y normativo y de estímulo a los gobiernos, organizaciones y personal de salud, para promover y apoyar la atención de la salud con inclusión de la medicina tradicional. Asimismo, en la conferencia de AlmaAta (Kazajistán), celebrada en 1978, se acordó impulsar la documentación y evaluación científica de las plantas utilizadas en la medicina tradicional, abriendo las puertas al diálogo entre la medicina tradicional y la moderna, sobre la base de que las prácticas peligrosas se eliminarian y solo se promovería lo que fuese seguro y eficaz. (Bermúdez, 2005; OPS, 1991; Albornoz, 1993; Akerele, 1993). Por su parte, la Organización de las Naciones Unidas para la Alimentación (FAO), resalta que los conocimientos de los pobladores indigenas "han contribuido al descubrimiento de alrededor de tres cuartas partes de los medicamentos de origen vegetal que se emplean ampliamente en el mundo desarrollado". (Bocanegra, 2011; Mendocilla, 2001; Baltodano, 2006).

En tal sentido, para la OMS, la medicina tradicional comprende diversas prácticas, enfoques, conocimientos $\mathrm{y}$ creencias sanitarias que incorporan medicinas basadas en plantas, ani- 
males o minerales, en terapias espirituales, en técnicas manuales y en ejercicios aplicados de forma individual o en combinación para mantener el bienestar; además de tratar, diagnosticar y prevenir las enfermedades. (OMS, 2002; Oblitas, 2013). En cuanto al contexto latinoamericano, cuando se hace referencia a la medicina tradicional, se refiere a la medicación, materiales elaborados, preparados y productos a base de hierbas que contienen ingredientes activos de plantas $u$ otros materiales vegetales o combinaciones de las mismas. (Bocanegra, 2011).

Es por ello que, desde el punto de vista sociocultural, se promueve que todo trabajador de salud comunitaria debe tener presente en el momento del desarrollo de sus actividades, lo siguiente con respecto a la medicina tradicional (Chifa, 2010):

- respetarlas como expresión de cultura popular;

- recuperar esas prácticas para utilizar y mostrar las riquezas y variedad de respuestas en salud;

- sistematizar y conceptualizar teóricamente esas prácticas para su discusión, investigación, divulgación, aprovechamiento colectivo y enseñanza.

Entre las razones que se señalan para el uso de plantas medicinales destaca su carácter natural, abundancia, facilidad de adquisición, efectividad, ausencia de efectos adversos, bajo costo, tradición familiar o recomendación de terceros experimentados. También se menciona la curación lenta pero segura, la confianza, la ca- pacidad de curar todo, la ineficacia de los medicamentos y el no necesitar medicinas ni prescripción médica. ((Bocanegra, 2011; Viganó, 2007; Bussmann, 2007).

En cuanto al uso terapéutico de las plantas medicinales, este está generalmente referido al tratamiento y prevención de problemas digestivos (diarrea, dolor abdominal, parasitosis y hepatopatías); problemas respiratorios (catarros, dolor de garganta, rinitis); problemas nerviosos (nerviosismo, insomnio, estrés); problemas circulatorios (enfermedades del corazón, hipertensión, arterias y venas); problemas endocrinos (diabetes); problemas genitourinarios; problemas osteomusculares; problemas dérmicos y otros, como sobrepeso, astenia, menopausia, dolor de cabeza, caída de cabello e inflamaciones. (Bocanegra, 2011; Viganó, 2007; Llorach, 2007; Anaya, 2015).

Entre las propiedades que poseen las plantas medicinales, se menciona principalmente su poder (Municipalidad de San Borja, 2014; Morales, 2014):

- Abortivo: interrumpe el embarazo o provoca la expulsión del feto antes de tiempo.

- Afrodisíaco: estimula o excita la actividad sexual.

- Alucinógeno: provoca alucinaciones modificando las percepciones.

- Amargo: ver antianoréxico.

- Analgésico: calma o elimina el dolor, ya sea de cabeza, muscular o artrítico.

- Antiácido: actúa contra la acidez estomacal. 
- Antianoréxico: estimula las secreciones gástricas, ayudando a la digestión y favoreciendo el apetito; también se les denomina aperitivas o amargo.

- Anticancerígeno: impide el desarrollo, crecimiento o proliferación de células tumorales malignas.

- Anticonceptivo: impide la fecundación y por tanto el embarazo.

- Antidiabético: reduce los niveles de glucosa en la sangre.

- Antidiarréico: corta la diarrea.

- Antiemético o antivomitivo: evita o detiene los vómitos o náuseas.

- Antiespasmódico: alivia o calma los espasmos (movimientos involuntarios de los músculos de intestinos, uréteres, vesícula, que acusan los cólicos).

- Antigonorreíco: sana la gonorrea o blenorragia.

- Antihelmíntico, vermífugo o vermicida: expulsa o destruye los parásitos intestinales.

- Antihemorrágico: véase hemostático.

- Antihemorroidal: resuelve las hemorroides o calma su dolor.

- Antihistamínico: bloquea los síntomas de las alergias (congestión, picazón de los ojos, estornudo y goteo de la nariz, así como la comezón de la urticaria).

- Antiinflamatorio: combate la inflamación o hinchazón de los tejidos orgánicos.

- Antimicrobiano: extermina microbios causantes de enfermedades y ayudan a fortalecer los mecanismos de defensa del organismo.
- Antineurálgico: sana las neuralgias y dolores ocasionados por la irritación de los nervios periféricos.

- Antiofidico: antídoto contra la picadura de serpiente venenosa.

- Antipirético: disminuye la fiebre.

- Antiséptico: sustancia que se aplica sobre la piel para reducir la posibilidad de infección, sepsis o putrefacción.

- Antiséptico vaginal: impide la infección en la vagina y las partes sexuales de la mujer.

- Antitérmicas: bajan la temperatura.

- Antitusígeno o béquico: combate o calma la tos.

- Aperitivo: ver antianoréxico.

- Astringente: astringe, aprieta, estrecha, contrae o retiene alguna sustancia de los tejidos orgánicos. Disminuye la secreción de las mucosas, retrae y endurece los tejidos orgánicos. Usualmente se refiere a que disminuye la secreción mucosa, aumenta la absorción de líquidos por parte de los intestinos, disminuyendo la diarrea.

- Balsámico: alivia la irritación de la garganta y reconforta las vías respiratorias.

- Calmante: se refiere especialmente a las plantas con efectos sedantes y a las que disminuyen o hacen desaparecer el dolor $\mathrm{u}$ otro sintoma molesto.

- Carminativo: calma la inflamación de las paredes intestinales y permite la eliminación de gases 
del tubo digestivo. Provoca la expulsión de los gases intestinales y alivia la tensión gastrointestinal.

- Cicatrizante: estimula el crecimiento de los tejidos en las heridas, favoreciendo la cicatrización.

- Depurativo: depura, purifica o limpia los humores (líquidos) del cuerpo, sobre todo la sangre a través del sudor o la orina.

- Descongestionante nasal: destapa la nariz.

- Desinfectante: véase antiséptico.

- Desintoxicante de alcohólicos: ayuda a que las personas alcohólicas dejen el hábito de beber alcohol.

- Digestivo: véase sudorífico.

- Diurético: aumenta la producción y eliminación de orina.

- Emenagogo: provoca la menstruación.

- Emoliente: tiene acción antiácida y protege a los tejidos irritados o inflamados.

- Estimulante: combate los efectos de la fatiga.

- Estimulante del parto: que activa o estimula el trabajo del parto facilitando la salida del feto.

- Estimulante del sistema digestivo: induce el apetito y producción de jugos digestivos.

- Eupéctico: ver antianoréxico.

- Expectorante: permite la eliminación de mucosidades alojadas en vías respiratorias.

- Galactogogo: incrementa la secreción láctea durante la lactancia.

- Hemostático: favorece o provoca la coagulación de la sangre y detiene la salida de sangre en las hemorragias.

- Hepatoprotector: tiene la función de proteger el hígado para que funcione adecuadamente.

- Hipoglucemiante, antidiabético o antiglucemiante: reduce la cantidad de glucosa (azúcar) en la sangre y orina de los diabéticos.

- Insecticida: capaz de matar insectos.

- Laxante (Purgante): acelera la evacuación de las heces y mejora el tránsito intestinal. Estimula la evacuación intestinal en caso de estreñimiento.

- Pediculado: mata los parásitos que producen la sarna o rasca rasca.

- Purgante: que purga; es decir, que limpia el vientre de las sustancias que le son dañinas.

- Purificador de la sangre: véase depurativo.

- Relajante: relaja la tensión muscular y nerviosa.

- Soporífero: facilita el sueño.

- Sudorífico: provoca sudoración.

- Tranquilizante: ayuda a reducir y controlar estados de nerviosismo, ansiedad e inquietud

De otro lado, se ha reconocido doce países megadiversos, que tienen el $70 \%$ de la flora mundial. Dentro de ellos, cinco pertenecen a América Latina: Brasil, Colombia, Ecuador, México y Perú. Los otros países son Australia, China, India, Indonesia, Madagascar, Malasia y Zaire. (Diario Expreso, 2015b). Aunque no existen datos precisos para evaluar la extensión del uso 
global de plantas medicinales, la OMS ha estimado que más del $80 \%$ de habitantes en países en desarrollo recurre frecuentemente a la medicina tradicional para satisfacer sus necesidades de atención primaria de salud, y que gran parte de los tratamientos tradicionales implica el uso de extractos de plantas o sus principios activos. Esta es una de las razones por la que la OMS promueve cada vez con más intensidad el estudio de las plantas utilizadas en la medicina tradicional herbolaria. (Bermúdez, 2005; Bocanegra, 2011).

A pesar del aumento de los estudios en estas plantas, los datos disponibles señalan que apenas el 15\% han sido estudiadas en su potencial medicinal para un efecto específico, de un aproximado de 250 a $500 \mathrm{mil}$ especies existentes en el planeta, de las cuales solo el $10 \%$ se puede considerar medicinales. También se estima que el 20\% de las prescripciones médicas contienen productos a base de plantas. (Lock, 2009).

Actualmente, en países como Hungría, Polonia, Alemania e Inglaterra, las investigaciones terapéuticas están adquiriendo un empuje insospechado. Asimismo, los norteamericanos desde hace años envian investigadores a distintos países para el estudio de las plantas consideradas en cada lugar como medicinales; estudio llamado etnofarmacognosia, y que se basa en el conocimiento de origen popular o de transmisión oral. (Diario Expreso, 2015c).

\section{El Perú}

Se caracteriza por una gran biodiversidad vegetal y una cultura tradicional de uso de plantas con propósitos medicinales muy arraigada en los pueblos andinos y amazónicos. Sin embargo, esta medicina tradicional no ha logrado formalizarse como ha ocurrido en algunas culturas asiáticas, como la India, China y Corea. Uno de los problemas es que la transmisión del conocimiento tradicional indígena en el Perú es por vía oral y poco o nada aparece registrado en textos confiables. El desconocimiento de la escritura es una de las causas de la ausencia de mucha información, aunque parte de ello ha sido incorporado en documentos escritos durante la conquista española a partir del siglo XVI, particularmente a través de los cronistas. (Gonzales, 2011; Calixtos, 2006).

Asimismo, se estima en 25 mil las especies existentes, aunque algunos científicos calculan que esta cifra puede ser duplicada. Aproximadamente 4 mil especies tienen diversos usos en la alimentación, salud, cosmética, tintura, aromatizantes y saborizantes, entre otros. (Gonzales, 2011; Calixtos, 2006). La participación que tiene el país en el mercado mundial de productos naturales solo es de $0,02 \%$. Solo se utilizan unas 1400 especies con propiedades medicinales de uso popular, de las cuales solo un pequeño porcentaje de estas y sus derivados se comercializan dentro y fuera del país. (Brack Egg, 1999; Pamo-Reyna, 2009). 
Las razones para el uso de plantas medicinales obedecen a la riqueza y variedad en especies vegetales y a la tradición existente sobre su empleo desde el periodo preinca, que ha persistido hasta la actualidad. El uso de plantas medicinales coexiste con la medicina occidental, especialmente en el primer nivel de atención, donde su uso está orientado a prevenir $\mathrm{y}$ aliviar diversos problemas de salud (Baltodano, 2006). Además, según las encuestas realizadas a expendedores y compradores, el uso y manejo de las especies medicinales nativas están relacionadas y arraigadas al conocimiento ancestral transmitido de padres a hijos, el cual es mayormente expresado en las zonas rurales. (Huamantupa, 2011).

Se debe también tener en cuenta que en el Perú un tercio de las personas vive en pobreza o pobreza extrema, por lo que no cuentan con los medios económicos necesarios y no tienen acceso a los servicios de salud, lo cual los conduce a recurrir a la medicina tradicional por ser más accesible y barata que los productos farmacéuticos. (Bermúdez, 2005). Asimismo, en las grandes ciudades viven personas que en su mayoría son migrantes o hijos de migrantes de la costa, sierra y selva, quienes están culturalmente más asociados al consumo de productos naturales para el tratamiento de problemas relacionados a la salud. Estos conocimientos han sido adquiridos de sus ancestros, brujos o chamanes y/o personas de sus comunidades. (Lock, 2009).
Cabe indicar en el año 2007 se realizó una cumbre internacional organizada por el Colegio Médico del Perú, conocida como La Declaración de Lima, la cual reconoce, entre otros puntos, la importancia de la medicina tradicional y recomienda su armonización y articulación con los sistemas de salud oficiales de cada país. La medicina occidental y la tradicional no son excluyentes, sino coexisten, dependiendo de la disponibilidad de los servicios de salud. En el Perú, el tema ha sido trabajado mediante varios estudios de etnobotánica realizados en diversas poblaciones; sin embargo, no se han encontrado estudios sobre la frecuencia de empleo de plantas medicinales ni en la población en general ni entre aquellos que acuden a hospitales del sistema oficial de salud, especialmente al tercer nivel de atención, donde existe una mayor oferta de servicios de salud, que incluye medicamentos, terapias y servicios disponibles. Esto ocurre principalmente en las zonas netamente rurales. (Oblitas, 2013).

\section{Conclusiones}

En la actualidad, a pesar de existir la medicina convencional o científica, muchas personas practican y consumen productos naturales para hacer frente a enfermedades o problemas de salud. En muchos casos, la medicina occidental y el sistema de salud no aceptan el uso de productos naturales como una alternativa o complemento al tratamiento farmacológico, tal vez por el desco- 
nocimiento de sus beneficios o por no tener confianza en su efectividad para combatir una enfermedad. (Lock, 2009).

Asimismo, es conveniente que su uso sea racional, para no convertirla en un arma de doble filo que atente contra la salud de quienes la utilizan. Por ello, antes de consumir cualquier tipo de planta, es indispensable consultar a un experto o conocedor. Sobre todo, deben tener cuidado personas con determinadas características, o que sufren alguna enfermedad o problema de salud. (Diario Expreso 2015a). Además, la falta de controles adecuados en la fabricación así como en la comercialización, han permitido la falsificación del contenido de muchos productos herbarios, en los cuales se ha encontrado carga microbiana (equivalente a contaminación fecal). (Sáenz, 2003).

Cabe destacar que en los últimos años la investigación científica de las plantas medicinales ha resurgido con inusitado interés, partiendo del hecho de que gran parte de la población de muchos pueblos recurre a la medicina tradicional como única fuente para resolver sus problemas de salud. (Alvarado, 2007). En tal sentido, las Facultades o Escuelas de Medicina deberian incluir en sus currícula, la enseñanza de la medicina tradicional, sus productos, en qué casos se puede aplicar y las contraindicaciones de uso. Estos conocimientos, debidamente sistematizados, podrian contribuir a resolver, en parte, los problemas de salud de la población menos favorecida y más alejada de los centros de tratamiento formal, cuyas posibilidades de curarse son limitadas por el alto costo de los fármacos modernos. (Mejía, 2000).

Finalmente, no puede dejar de mencionarse que algunos médicos se oponen al empleo de los remedios y los métodos de la medicina popular, debido a que algunas plantas medicinales no han mostrado las propiedades que les atribuye la experiencia popular e, incluso, algunas han resultado peligrosas porque no existe una base científica que sustente sus beneficios para la salud. (Diario Expreso, 2015b). 


\section{REFERENCIAS BIBLIOGRÁFICAS}

Akerele O. (1993). "Las plantas medicinales: un tesoro que no debemos desperdiciar". Foro Mundial de la Salud, 14, 390-395.

Albornoz, A. (1993). Medicina Tradicional Herbaria (Guia de Fitoterapia). Instituto Farmacoterápico Latino. Caracas, Venezuela.

Anaya Hurtado, M.J., Musayon Aranda, C.A. y Onton Vargas, E.E. (2015) "Conocimiento y uso de plantas medicinales en los pobladores del Distrito de Picsi (Chiclayo)". Obtenido el 15-06-2015 de http://en.scientificcommons. org/32278250

Alvarado Chávez, B. (2007). "Plantas medicinales de la Cordillera Negra". Rev Acad Peru Salud, 14(2), 52-63.

Baltodano Alfaro, E. (2006). "Las plantas medicinales y sus efectos curativos". Penochid, 2(2), 40-41.

Bermúdez, A., Oliveira Miranda, M.A. y Velázquez D. (2005). "La investigación etnobotánica sobre plantas medicinales: una revisión de sus objetivos y enfoques actuales". INCI, 30(8), 453-459.

Bocanegra García, L.M., Bocanegra Díaz, F.A. y Mostacero León, J. (2011). "Efectividad de la medicina herbolaria y su impacto en la calidad de vida de los pobladores de Curgos, Perú”. UCV-Scientia, 3(1), 23-34.

Brack Egg, A. (1999). Diccionario Enciclopédico de Plantas Útiles en el Perú. Cusco: Centro Bartolomé de las Casas.

Bussmann, R.W., Sharon, D. y López, A. (2007). "Blending Traditional and Western Medicine: Medicinal plant use among patients at Clinica Anticona in El Porvenir, Peru". Ethnobotany Research \& Applications., 5, 185- 199.

Calixtos Cotos, M.R. (2006). "Plantas medicinales utilizadas en odontologia" (Parte 1), Kiru, 3(2), 80-85.

Chifa, C. (2010). "La perspectiva social de la medicina tradicional". Boletín Latinoamericano y del Caribe de Plantas Medicinales y Aromáticas, 9(4), 242-245.

Diario Expreso. (2015a). "Plantas y salud". En: El Súper Libro de la Medicina Natural, Fascículo 1, Lima: 001.

Diario Expreso. (2015b). "La fitoterapia”. En: El Súper Libro de la Medicina Natural, Fascículo 1, Lima, 004.

Diario Expreso. (2015c). "Estudios científicos”. En: El Súper Libro de la Medicina Natural, Fascículo 2, Lima, 006.

García Cáceres, U. (2004). "Farmacopea peruana". En: Enciclopedia Temática del Perú. Capítulo 12 Salud, Editora El Comercio, Lima, 150-168.

Gómez-Veloz, A. (2002). Plant use knowledge of the Winikina Warao: The case for questionnaires in ethnobotany. Econ. Bot., 56, 231-242.

Gonzales, G.F. (2011). "Maca, producto de bandera del Perú: de la tradición a la ciencia”. Rev Acta Andina, 11(1), 56-67. 
Huamantupa Chuquimaco, I., Cuba, M., Urrunaga Soria, R.M., Paz Apaza, E., Ananya Bellido, N., Callalli, M., Pallqui Camacho, N.C. y Coasaca, H. (2011). "Riqueza, uso y origen de plantas medicinales expendidas en los mercados de la ciudad del Cusco". Rev. Peru. Biol, 18(3), 283-291.

Joshi, A. y Joshi K. (2000). "Indigenous knowledge and uses of medicinal plants by local communities of the Kali Gandanki Watershed Area, Nepal". J. Ethnopharmacol, 73, 175-183.

Katewa, S., Chaudhary, B. y Jain A. (2004). "Folk herbal medicines from tribal area of Rajastan, India”. J. Ethnopharmacol, 92, 41-46.

Llorach, C., Chen, C., González, E., Hernández, A. y Rodríguez, Y. (2007). "Uso de plantas medicinales en adultos que acuden a una unidad de atención primaria de Panamá". Archivos de Medicina Familiar y General., 4(1), 15-18. Lock Sing, O. (2009). "Flora andina y amazónica: un aporte a su conocimiento químico”. Acad. Nac. Cienc. Bol, (3), 34-42.

Mejía, K. y Rengifo, E. (2000). Introducción. En: Plantas Medicinales de Uso Popular en la Amazonía Peruana, Segunda Edición Corregida y Aumentada, Agencia Internacional de Cooperación Española (AECI) - Instituto de Investigaciones de la Amazonía Peruana (IIAP), Iquitos, 8-9.

Mendocilla M. y Villar López, M. (2001). "Monografias de plantas medicinales". En: Manual de Fitoterapia. EsSalud y Organización Panamericana de la Salud/Organización Mundial de la Salud, Lima, 91-345.

Morales Taipe, V. "Catálogo de Plantas Medicinales Estudiadas en la Facultad de Farmacia y Bioquímica de la UNMSM (Período: 1924 - 1986)”. Municipalidad de San Borja. (2014). Glosario. En: Guía de Salud y Primeros Auxilios, Edición Especial para los Vecinos de San Borja, Lima, 131-133.

Oblitas Zanabria, G.S., Hernández Córdova, J.G., Chiclla, A., Antich Barrientos, M.K., Ccorihuamán Cusitito, J.L. y Romaní Romaní, FR. (2013). "Empleo de plantas medicinales en usuarios de dos hospitales referenciales del Cusco, Perú". Rev Peru Med Exp Salud Publica, 30(1), 64-68.

Oficina Panamericana de la Salud. (1991). "Plantas medicinales como fuente de medicamentos". Boletín de la Oficina Sanitaria Panamericana, 11(2), 175176.

Organización Mundial de la Salud. (2002). Estrategia de la OMS sobre medicina tradicional 2002-2005. Ginebra.

Pamo-Reyna, O.G. (2009). "Características de los trabajos publicados sobre las propiedades de las plantas en revistas médicas peruanas”. Rev Peru Med Ex Salud Publica, 26(3), 314-323.

Pan American Health Organization. (2004). "Recomendaciones de la OMS para gobiernos y consumidores acerca del uso de los tratamientos tradicionales". Rev Panam Salud Pública., 16(3), 218-221. 
Prance, G. (1991). "What is the ethnobotany today?". J. Ethnopharmacol, 32, 209-216.

Sáenz Campos, D. (2003). "Medicamentos, plantas medicinales y productos naturales”. Fármacos., 16(1-2), 13-20.

Tabuti, J.R., Lye, K.A. y Dhillion, S. (2003). "Traditional herbal drugs of Bulamogi, Uganda. Plants, use and administration". J. Ethnopharmacol, 88, 19-44.

Viganó, J., Viganó, J.A. y Araujo da Cruz-Silva, C.T. (2007). "Utilização de plantas medicinais pela população da região urbana de Três Barras do Paraná". Acta Sci. Health Sci.,29(1) ,51-58.

Villar López, A. y Mendocilla Risco, J. (2001). "Farmacología de las plantas medicinales". En: Manual de Fitoterapia. Lima: EsSalud y Organización Panamericana de la Salud/Organización Mundial de la Salud. 65. 